\title{
MARS EXPRESS LITHIUM ION BATTERIES PERFORMANCE ANALYSIS
}

\author{
G. Dudley ${ }^{(1)}$, R. Blake ${ }^{(2)} \&$ L. Lucas ${ }^{(3)}$ \\ (1)ESA/ESTEC Keplerlaan 1, 200 AG Noordwijk, the Netherlands (retired),Email: geoff.dudley@planet.nl \\ (2) SCISYS UK, Methuen Park, Chippenham, SN14 0GB United Kingdom, Email: rick.blake@scisys.co.uk \\ (3) LSE Space GmbH, Robert-Bosch-Straße 7, 64293 Darmstadt, Germany,Email: luke.lucas@esa.int
}

\begin{abstract}
Now more than 12 years in orbit, Mars Express battery telemetry during some of the deepest discharge cycles has been analysed with the help of the ESTEC lithium ion cell model. The best-fitting model parameter sets were then used to predict the energy that is expected to be available before the battery voltage drops below the minimum value that can support the power bus. This allows mission planners to determine what future power profiles could be supported without risk of entering safe mode. It also gives some more insights into the ageing properties of these batteries.
\end{abstract}

\section{INTRODUCTION}

After Proba-1, Mars Express, launched in June 2003 for an initial 4-year mission, is the spacecraft with the longest duration in orbit that relies upon lithium ion batteries to provide main-bus energy storage. Because of an error in the power subsystem discovered soon after launch, the maximum available energy available from the solar arrays was reduced to $72 \%$ and power management became particularly critical in order to permit planned science operations without the risk of entering safe mode.

The spacecraft carries 3 identical batteries each with a 6s 16p configuration, (16 parallel-connected strings of 6 Sony $18650 \mathrm{HC}$ cells in series). Each battery is connected to a separate battery charge-discharge regulator, controlled such as to ensure equal current sharing between batteries. Telemetry channels provide battery voltage, temperature, charge current and discharge current. The high precision of the measurements after calibration is shown in Tab. 1.

\begin{tabular}{|c|c|c|}
\multicolumn{4}{|c|}{ Table 1. Battery telemetry details } \\
\begin{tabular}{|c|c|c|}
\hline Parameter & $\begin{array}{c}\text { Calibration } \\
\text { Resolution }\end{array}$ & Frequency \\
\hline Voltage & $0.0075 \mathrm{~V}$ & $1 / 32$ seconds \\
\hline Current & $0.0026 \mathrm{~A}$ & $1 / 32$ seconds \\
\hline Temperature & $0.0267{ }^{\circ} \mathrm{C}$ & $1 / 32$ seconds \\
\hline
\end{tabular}
\end{tabular}

The batteries cannot be discharged individually to determine their useful capacity so the problem is to predict how much energy would be available under a given power and ambient battery temperature profile using telemetry date from discharge-charge cycles with relatively shallow depths of discharge.

Several battery models of varying complexity have been used to assess battery state of health using telemetry data from the longest eclipses [1]. Comparison of ground test battery performance data with early in-flight eclipse data using the lithium ion battery model developed at ESTEC [2] was reported at the 2006 NASA Aerospace Battery Workshop [3]. Despite some difficulties, which will be described later, it was possible to arrive at a reasonable estimate of battery energy capacity loss. Now, 10 years later and after improvements in the model and the availability of ground test data on aged batteries this analysis is revisited and extended to include recent telemetry.

\section{MARS EXPRESS MISSION}

After a six-month cruise plus orbit insertion phase, Mars Express entered into an elliptical polar orbit around Mars in December 2003. There are approximately 3.5 orbits per day and the spacecraft is subjected to two eclipse seasons each Mars year (a season roughly every 10 months). The number of eclipses and maximum eclipse duration varies from one season to another as can be seen from Fig. 1. During eclipses the spacecraft bus has to be maintained by the batteries whilst at other times the spacecraft is in continuous sunlight but the batteries are sometimes required to supplement power from the solar arrays.

At the time of writing Mars Express has just entered the $2016 / 2017\left(16^{\text {th }}\right)$ eclipse season. At the end of 2015, the total number of discharge cycles was approximately 33605 , of which the majority (26409) were less than $0.25 \mathrm{Wh}$. There were 4168 discharges in the range $25-$ $100 \mathrm{Wh} /$ battery and 3022 discharges in the range $100-$ $250 \mathrm{Wh}$ - more than the manufacturer's qualification limits for the nominal mission of 2397 and 1531 respectively, but the batteries are still well below the 510 limit for discharges above $250 \mathrm{Wh}$.

The mission is currently approved until end 2018, with a decision on a further extension to $2019 / 20$ expected in autumn 2016. 


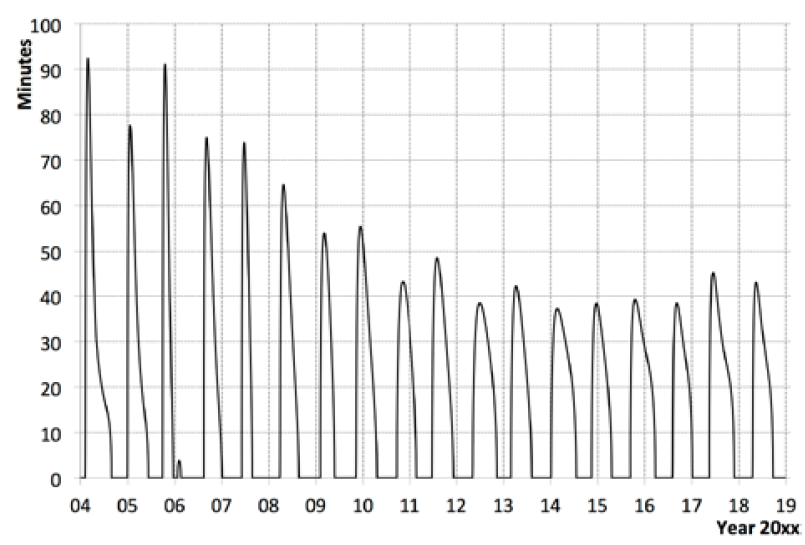

Figure 1. Eclipse duration versus date 2004-2018

The performance of the batteries is a key part of any long term planning particularly with regard to the ability of the spacecraft to operate and survive future eclipse seasons. Preliminary analysis of the next two eclipse seasons in 2016 and 2017 is already underway and while the 2016 season appears relatively benign in terms of eclipse duration and power availability (season coincides with perihelion) the 2017 eclipses presents significant challenges as the they are longer, power availability will be lower and more heater power will be required as Mars passes through aphelion.

From analyses of the longest eclipses it was realised that deeper discharges would be needed to get more reliable battery state of health information. To this end six special deep discharge tests (DDTs) were carried out between 2008 and 2012. Outside of eclipse seasons, the solar arrays were deliberately pointed away from the sun in order to discharge the batteries until the first reached $21 \mathrm{~V}$ or a time limit expired after which the arrays were automatically restored to sun-pointing. Since 2012 these have not been continued for fear of increasing the stress on the batteries.

\section{APPLICATION OF CELL MODEL TO BATTERY TELEMETRY}

In the ESTEC model for the ABSL $18650 \mathrm{HC}$ cell [2] the cell voltage is determined by:

1. The EMFs of the positive and negative electrodes as a function of state of charge. The cell EMF is the difference between these two.

2. The variation in cell EMF with temperature as a function of state of charge

3. The cell internal resistance (due to electrolyte, current- collectors and electrode-electrolyte interfacial resistances), independent of state of charge but a strong function of temperature

4. Diffusion polarisation in the electrodes, independent of state of charge but a strong function of temperature
5. EMF hysteresis (the EMF versus state of charge curve differs slightly between charge and discharge), a strong function of state of charge but independent of temperature

Tests on cycled cells [4] show that 1,2 and 5 are not affected significantly by ageing until very close to end of life. The cell capacity decreases but the EMFs of the electrodes are simply 'compressed' in proportion to their diminishing capacity. The extent to which the two electrodes lose capacity is however different and the relative state of charge between them at the reference state of $4.2 \mathrm{~V}$ does change with ageing. Item 3 increases strongly with ageing whereas the item 4 increase is modest. It was found that ageing could be incorporated into the model simply by modifying the values of 6 parameters:

1. Internal resistance [Res_factor] - amount by which the beginning of life (BoL) resistance has to be multiplied

2. Diffusion resistance [Res_factord] - as above for diffusion resistance

3. Positive electrode capacity factor [Cfp] - amount by which BoL positive electrode capacity has to be multiplied

4. Negative electrode capacity factor [Cfn] - as above for the negative electrode

5. Positive state of charge (SoC) at a cell voltage of 4.2 [Ahpinit] - deviation from BoL value of 0

6. Negative SoC at a cell voltage of 4.2 [Ahninit] - as above for negative electrode

Characterising a cycled cell therefore reduces to the determination of the values of the 6 above parameters. Because of the lack of characterisation data for aged cells at temperatures around -5 to $+5^{\circ} \mathrm{C}$ that the Mars Express batteries experience, it has had to be assumed that at any given point in time the changes to the parameters due to ageing are the same at all temperatures.

\subsection{Model update}

Best-fit values of Res_factord found in earlier reported MEX telemetry analyses [3] where the batteries are cold were in the region of 0.5 rather than 1 , whereas in ground tests at $20^{\circ} \mathrm{C}$, expected values near to 1 were found. The explanation for this only became evident following recent and precise current interruption resistance measurements on other lithium ion cell types [5]. These showed that while the interfacial resistance (activation polarisation) contribution to the cell internal resistance $R_{i}$ is negligible at $20^{\circ} \mathrm{C}$ and above, it can become significant at lower temperatures. Moreover it typically takes several hundred milliseconds to approach a steady value because of the large double-layer capacity. At the time of the development of the 
$18650 \mathrm{HC}$ cell model (2004) the current interruption measurements used recorded the resistance after 70 to $100 \mathrm{mS}$. It is now realised that this would have been too short a time to capture the full interfacial resistance contribution at $0^{\circ} \mathrm{C}$ and thus would have lead to an under-estimation of $\mathrm{R}_{\mathrm{i}}$ at low temperatures. The diffusion resistance $R_{d}$ was calculated from the total steady state polarisation resistance $R_{\text {tot }}$ under constant current from the relation:

$$
R_{d}=1.5\left(R_{t o t}-R_{i}\right)
$$

In consequence it would be too large and result in the observed low values of Res factord. The model was updated by fitting $\mathrm{R}_{\mathrm{d}}$ to step cycle test data [2] at 0 and $20^{\circ} \mathrm{C}$ and deriving values at other temperatures from the Arrhenius slope defined by these two. $R_{i}$ is then obtained from Eq. 1 instead of the current interruption resistance measurements. Although a rather rough fix, dictated by the absence of better resistance measurements on the $18650 \mathrm{HC}$ cell, the updated model results in a reduction of the root mean square (RMS) fit errors with the Mars Express telemetry by some 5-20\%, and Res-factord parameter values much closer to 1 , showing that it is a definite improvement.

\section{BATTERY TELEMETRY ANALYSIS}

Since the cell model calculates the cell potential under a given imposed current profile, the simplest way of fitting the model parameters to the telemetry data is by imposing the battery telemetry current and temperature profiles on the model of a single cell scaled to the battery configuration, and comparing the resulting voltage prediction to the voltage telemetry. This implies that all cells in a battery have the same characteristics and state of charge. Since individual cell voltages are not monitored there is no other option, but ground tests have confirmed that cells in a battery do remain well matched until very near end of life [4]. The harness resistance from the battery to the point of battery voltage measurement was set to $0.0343 \mathrm{ohms}$.

The Berkeley Madonna application [6] used to implement the model includes a curve-fitting algorithm that can find a best-fit solution by varying an arbitrary number of parameters and running the model repeatedly until a minimum root-mean-square (RMS) error between measured and modelled battery voltage is reached. The algorithm (which is not publically documented) requires upper and lower limits to be set for each parameter together with two different initial guesses. However the best-fit parameter values that result differ depending on the limits and guesses that are chosen. This is because the algorithm only finds a 'local' minimum in the 6-dimensional parameter space. What is really wanted is the 'deepest' or 'global' minimum. Like the 'travelling salesman' problem this is a so-called 'NP-hard' problem for which there is no known way of finding with certainty this global minimum. Consequently it is necessary to run the curve-fitting algorithm repeatedly with different sets of limits and guesses and to assume that the ones with the smallest RMS errors are close to the global minimum. This is very processor - intensive since each current and temperature dataset consists of several thousand time value pairs and the curve fit routine for each set of guesses and limits runs the model typically $300-500$ times, taking several minutes on an average desktop computer. An auto step size integration (between 0.036 and 3600 seconds) was chosen ( $5^{\text {th }}$ order Runge-Kutta) with a tolerance of 1E-7. and the curve-fitting tolerance set to 1E-6. An 8-electrode slice model was used which is a good compromise between precision and compute speed [2].

Limits were always set well outside of plausible values, to avoid influencing the results, for example Cfp and Cfn upper limits were always $>1.1$ and Res_factor lower limits to 0.9 or less. Should any curve fit return a parameter value very close to a limit it was re-run using a wider limit. Occasionally implausible parameter vales were obtained such as $\mathrm{Cfn}>1$ or $\mathrm{C}_{0}>1.5$ and these fits were also not used. First and second guesses were varied randomly below and above expected parameter values. Small changes often produced a very different combination of parameter values. At other times they would result in an identical set of parameters.

\section{RESULTS}

Fig. 2 shows a typical model fit to the DDT6 telemetry. The RMS value of $0.03 \mathrm{~V}$ obtained corresponds to $5 \mathrm{mV}$ at cell level which is considered a good fit considering the number of factors that could degrade it amongst which are:

- Uncertainty and variability of actual cell internal temperatures.

- Cells no longer perfectly matched

- Difference between telemetry timestamps and instant of acquisition (important near major current changes)

- Possible current offset errors

To investigate the possible magnitude of the last item some test curve fits were performed with a current offset error as a $7^{\text {th }}$ parameter but this did not enhance the fits nor result in any consistent current error value so was dropped from subsequent analyses.

Fig. 3 shows the results for all 3 batteries from DDT 6 . Parameter values from the local minimum found for each curve fit are plotted against RMS error in $\mathrm{V}$ (battery level). It can be seen that there are a number of different combinations of parameters that give a similar quality of fit though there tends to be a cluster of points 
close to the best fit. These most likely correspond to minor minima in the vicinity of the global minimum caused by 'noise' in the data.

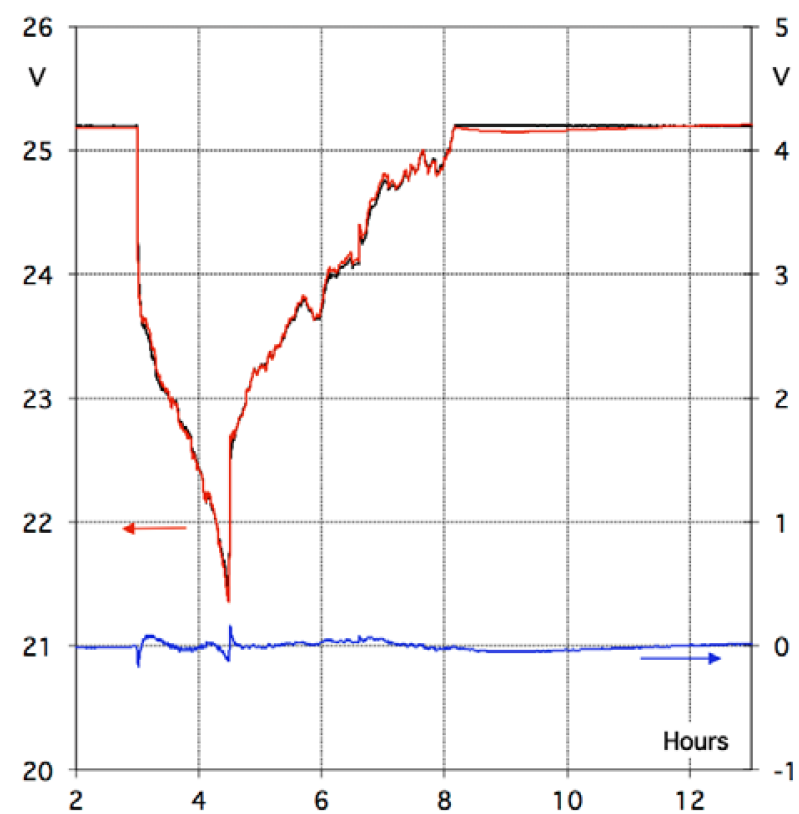

Figure 2. Telemetry (black), model (red), error (blue)

A still better fit that has not been found cannot be excluded but seems very unlikely. The general distribution of points for each parameter is very similar for all three batteries.

The battery cell capacity in $\mathrm{Ah}$ at zero current $\left(\mathrm{C}_{0}\right)$ is approximated by:

$$
C_{0}=1.487 \text { Cfn }+ \text { Ahninit - Ahpinit }
$$

$1.487 \mathrm{Ah}$ is the BoL capacity based on the model electrode EMF data. $\left(\mathrm{C}_{0}\right.$ is approximate because it counts the cell as charged when the positive electrode reaches the BoL EMF corresponding to a cell at $4.25 \mathrm{~V}$ and fully discharged when the negative electrode reaches the BoL EMF corresponding to a cell at $2.5 \mathrm{~V}$. In both cases the slope of EMF versus SoC of the other electrode is fairly flat so the error will be small).

Whilst the batteries appear to remain well matched to each other, battery 3 shows a slightly lower $\mathrm{C}_{0}$ value compared to 1 and 2 .

Tab. 2 shows details of the 9 cycles for battery 1 from different times in the mission that have been analysed with the aim of identifying how the battery has aged.

Table 2. Analysed Cycles

\begin{tabular}{|c|c|c|l|}
\hline $\begin{array}{c}\text { Cycle } \\
\text { date }\end{array}$ & $\begin{array}{c}\text { Ah } \\
\text { discharged } \\
\text { (cell) }\end{array}$ & $\begin{array}{c}\text { RMS fit } \\
\mathrm{mV} \\
\text { (battery) }\end{array}$ & \multicolumn{1}{|c|}{ Comments } \\
\hline $27 / 02 / 04$ & 0.599 & 18 & Eclipse season 1 \\
\hline $04 / 11 / 05$ & 0.717 & 22 & Eclipse season 3* \\
\hline $10 / 09 / 06$ & 0.438 & 27 & Eclipse season 4 \\
\hline $22 / 01 / 08$ & 0.713 & 30 & DDT 1 \\
\hline $26 / 06 / 09$ & 0.667 & 25 & DDT 3 \\
\hline $23 / 07 / 10$ & 0.678 & 25 & DDT 5 \\
\hline $07 / 05 / 12$ & 0.633 & 33 & DDT 6 \\
\hline $20 / 10 / 15$ & 0.345 & 25 & Eclipse season 15 \\
\hline $04 / 12 / 15$ & 0.351 & 24 & Eclipse season 15 \\
\hline
\end{tabular}

*Note: eclipse + shadowing by MARSIS payload boom.

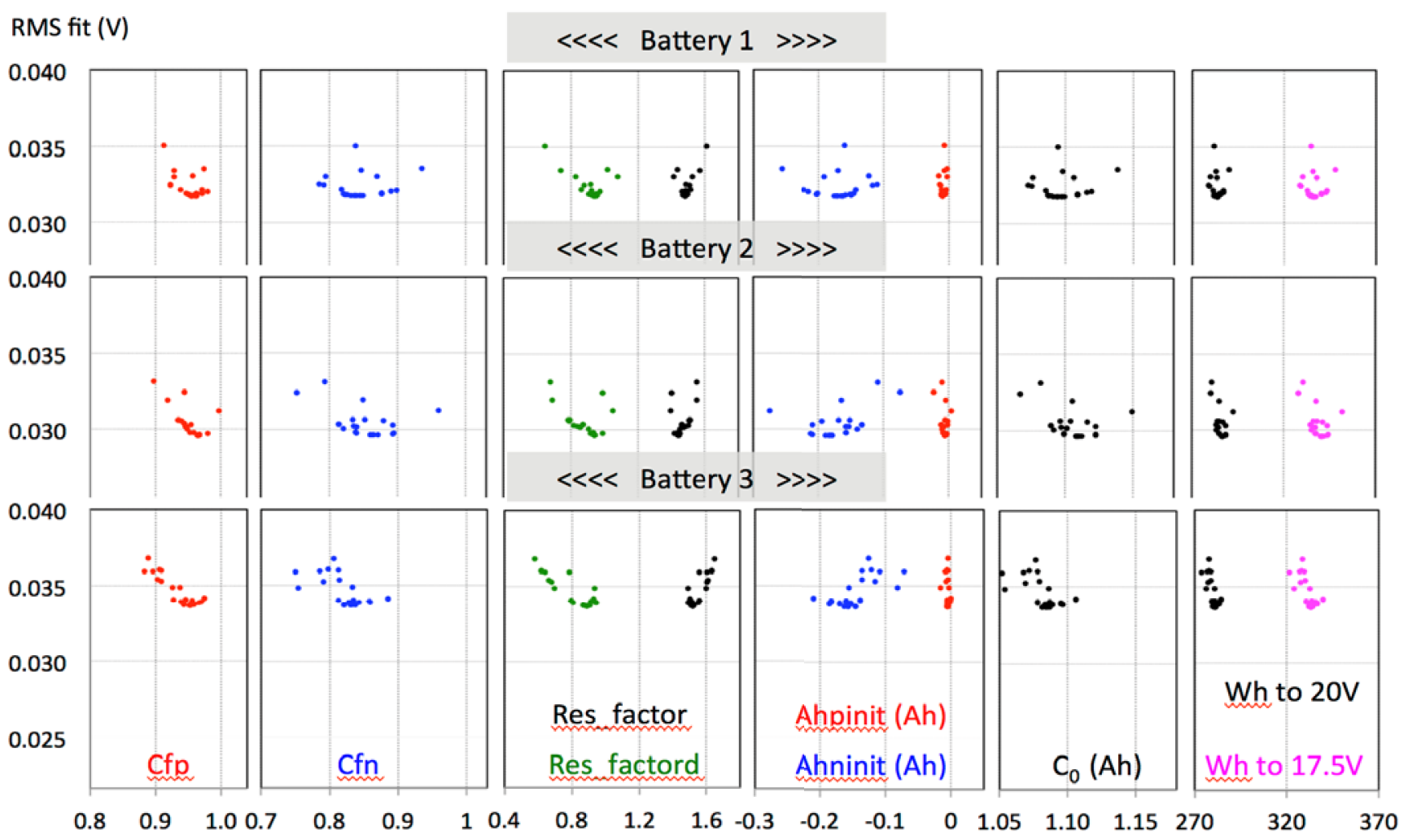

Figure 3. Curve fit results for DDT 6 (all batteries) 

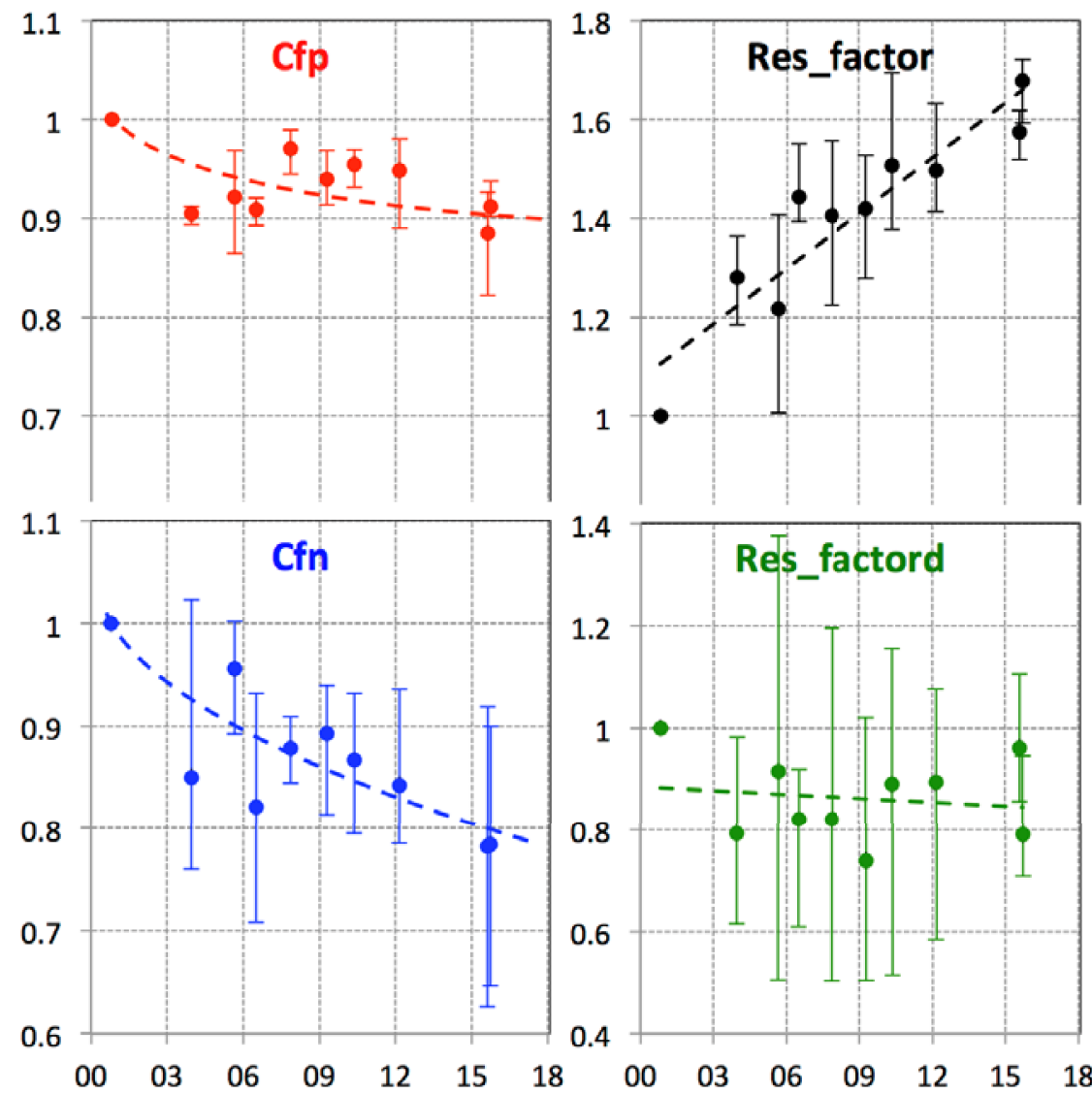

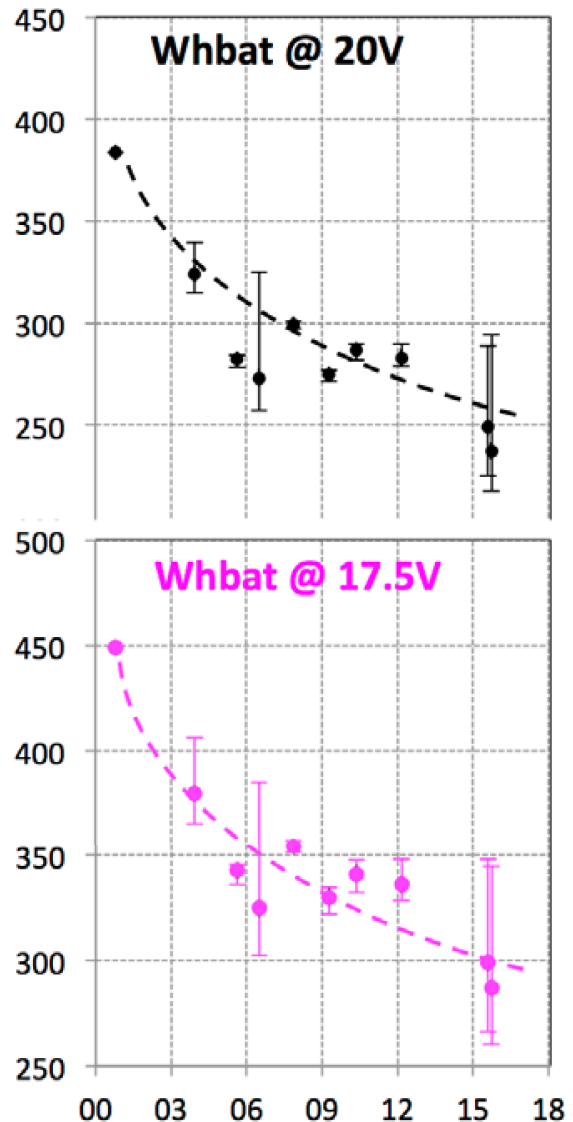

Figure 4. Average parameter values for battery las a function of date (20xx)

Fig. 4 shows the parameter values averaged over all curve fits (except those excluded for reasons described in section 4) plotted against date (last two digits of the year). BoL values are also shown positioned at 2001, the approximate time of manufacture of the cells. The error bars encompass the scatter in parameter values from the curve fits. The DDT results show a much smaller scatter than those from eclipse data because they were from deeper discharges. No curve fit from any DDT cycle gave rise to implausible parameter values but even so the scatter in $\mathrm{C}_{0}$ and $\mathrm{Wh}$ values in Fig.3 shows the danger of relying on the results of a single curve fit as was often done in the past. In contrast, curve fits from the shallower eclipse cycles gave much larger parameter value scatter. This is a direct result of the shape of cell electrode EMFs as a function of state of charge, shown in Fig. 5. EMF curves for a negative electrode which has lost $14 \%$ of its capacity and another which has BoL capacity but has lost 0.1 Ah of lithium are also shown. The EMF curves for these two possibilities only diverge significantly when the cell is more than 0.6 Ah discharged.

In curve fits from shallow cycles impossible parameter

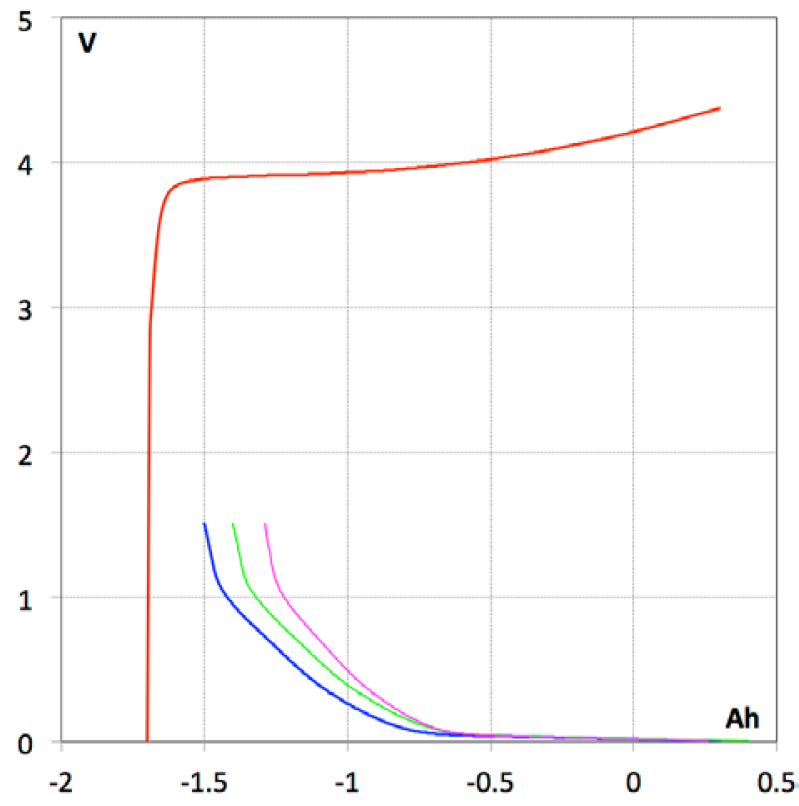

Figure 5. Sony 18650 HC electrode EMFs. Red: positive electrode; blue: negative electrode; green: negative electrode with 0.1 Ah lost lithium; violet: negative electrode with $14 \%$ capacity loss 
values now sometimes appear - for example in the last eclipse cycle the analyses with the smallest RMS error show values of $C$ fn well over 1 and values of $C_{0}$ well in excess of 1.47 . In the case of cycle $10 / 09 / 06$, only one third of the curve fits did not have such parameters and these were associated with smaller than expected $\mathrm{C}_{0}$ values. Others, rejected for producing $\mathrm{Cfn}$ values in the region of 1.01 to 1.3 produced $\mathrm{C}_{0}$ values in the expected region of 1.13 to 1.18 leading to a likely underestimate of available capacity.

From Fig. 5 it may appear surprising that the scatter in estimated capacity is not even larger since no discharge exceeded $0.713 \mathrm{Ah}$. That this is not the case is due to the combination of capacity and state of charge loss of the negative electrode. In addition, the state of charge of the part of the electrode nearest to the electrolyte (which contributes to the cell voltage) is lower than the average during discharge due to the lithium diffusion concentration gradient. Fig. 6 shows the equivalent $\mathrm{Ah}$ discharged of the electrode slices nearest to the electrolyte from the model fitted to the DDT 6 telemetry.

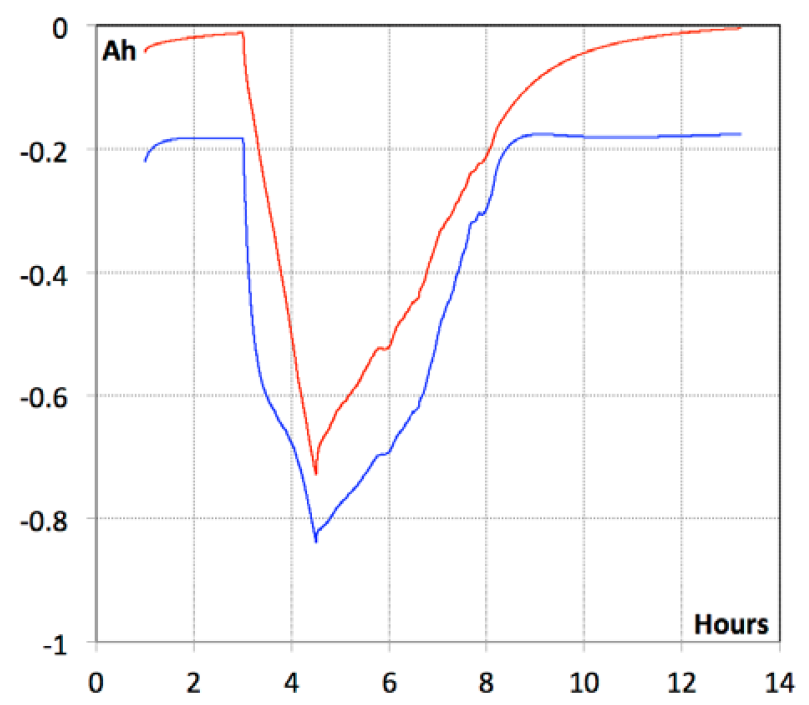

Figure 6. Ah discharged of electrode slices next to electrolyte during DDT 6. Red: positive electrode; blue: negative electrode;

Whereas the discharge during DDT 6 was -0.633 Ah (at cell level), the part of the negative electrode next to the electrolyte has discharged to $-0.82 \mathrm{Ah}(75 \%$ of aged cell capacity). Consequently the part of the EMF curve where the negative EMF starts to change rapidly is actually surpassed.

Res_factor and Res_factord show much scatter but high values for one correlate with low values for the other since the total steady-state resistance must be the same as shown in Eq. 1. Cfn and Ahninit also vary in a coupled fashion for the reasons explained earlier in connection with Fig. 5. As found in a ground tests [4], Res factor increases greatly with ageing due to the build up of reaction layers on the surface of the electrode active material particles. The activation and surface layer polarisation contributions also make an increasingly large contribution at low temperatures [5] and an increase with ageing is expected but has not been studied by the authors. In contrast Res factord has not increased much, behaviour also found in ground tests [4]. This might be expected since the parameter is determined by the rate of diffusion of lithium ions in the electrolyte in the pores of the electrodes and would be less affected by surface effects. The overall average value of around 0.85 rather than 1 for a $\mathrm{BoL}$ at $20^{\circ} \mathrm{C}$ is more likely due to the updated model still generating a slightly high diffusion resistance value at $0^{\circ} \mathrm{C}$ than indicating a real drop with time. Res_factor values correspondingly appear to be about 0.1 too high relative to BoL.

The results suggest that the positive electrode has lost capacity pre-launch and during transfer to Mars but not much since in orbit. It is less than was observed in ground tests at $20^{\circ} \mathrm{C}$ [5] so this may be explained by the relatively low temperatures in orbit. The slightly higher values found from the DDT analyses compared to the eclipse analyses is interesting but are thought more likely to be a shortcoming of the model rather than real. The negative electrode is down to around 80 to $90 \%$ of its BoL capacity, similar to ground tests. The results also indicate that this electrode has lost around 0.15 to $0.2 \mathrm{Ah}$ of lithium when fully charged due to reactions with the electrolyte, also similar to ground tests [5]. These two effects combine to reduce $\mathrm{C}_{0}$ down to $0.9 \mathrm{Ah}$ or $60 \%$ of BoL for the last cycle analysed.

\section{BATTERY PERFORMANCE PREDICTION}

$\mathrm{C}_{0}$ takes no account of the $60 \%$ increase in internal resistance that also has a strong effect on the usable capacity down to a specified cut-off voltage - the quantity that is much more relevant to spacecraft operations. To calculate this, parameter sets for each retained curve fit were fed into the model. A simple singe RC battery thermal model was used based on thermal capacity and resistance values that reproduced quite well the battery temperature telemetry. The starting temperature was set to $-2^{\circ} \mathrm{C}$, a typical value for the cycles analysed. A one- hour open-circuit followed by a $6 \mathrm{~A}$ constant current discharge was simulated. (this provides a margin over the average recent discharge currents of $4.48 \mathrm{~A}$ ). The initial electrode state of charge was adjusted so that the starting open-circuit voltage was 25.2. The Wh discharged to $20 \mathrm{~V}$ and to $17.5 \mathrm{~V}$ were extracted and are also plotted in Fig. 4. $20 \mathrm{~V}$ is the nominal battery discharge alarm surveillance (BDAS) limit and $17.5 \mathrm{~V}$ is the minimum settable value. The spread in Wh values between curve fits is less than that 
for $\mathrm{C}_{0}$ (not shown) and varies from as little as $1 \%$ for the best DDT to over $30 \%$ for the most recent eclipse cycles. Differences between successive DDTs which show increases in capacity as well as drops greatly exceed the smallest scatter values and it is not clear whether they are real and, for example, show possible recovery effects from lowering the voltage that batteries were held at between certain eclipses [7] or are due to shortcomings of the model.

Battery performance is enhanced at higher temperatures, so it is of interest to investigate what increase in useful Wh could be obtained if a battery deep discharge is started at say $+5^{\circ} \mathrm{C}$ instead of $-2^{\circ} \mathrm{C}$. In fact with a $6 \mathrm{~A}$ discharge there is only a gain of $6 \mathrm{Wh}$ at $20 \mathrm{~V}$ cut-off and $4.5 \mathrm{Wh}$ at a $17.5 \mathrm{~V}$ cut-off. This because by the time these voltages have been reached the battery has warmed by self-heating to over $16^{\circ} \mathrm{C}$ even when starting at $-2{ }^{\circ} \mathrm{C}$.

\section{CONCLUSIONS AND FURTHER WORK}

It has proved possible to fit the Mars Express battery 1 telemetry to the ESTEC battery model with RMS errors between 15 and $33 \mathrm{mV}$ (2.5 and $5.5 \mathrm{mV}$ at cell level), as good as one could expect and comparable to that obtained when fitting ground test data. Better cell resistance measurements on $\mathrm{BoL}$ cells at $0^{\circ} \mathrm{C}$ and $-10^{\circ} \mathrm{C}$, from which implementation of the temperature and SoC dependence of activation polarisation (5) could be added, could further improve the model accuracy.

This approach should be applicable to other spacecraft that use batteries containing lithium ion cells for which the ESTEC model parameters are available provided they deliver battery current, voltage and temperature telemetry of sufficient precision.

Despite the good fits there is a large scatter in parameter values depending on the initial settings of curve fits. Discharges of more than $0.6 \mathrm{Ah}$ at cell level are required to obtain reliable capacity values. This due to flat EMF versus Ah of the negative electrode which is the electrode that determines the cell capacity. The situation will be different for cells that use other than than hard carbon negative electrodes.

If this type of analysis is to be used routinely, there is a strong need for further automating the choosing of curve fit starting parameters and retrieval of the parameter values. With the absence of scripting capability in Berkeley Madonna, it is currently necessary to do this manually, which is very time-consuming even though each curve fit is quite fast. This is why Mars Express Batteries 2 and 3 have so far only been analysed for one cycle (DDT 6). An automated approach coupling Matlab with the EcosimPro implementation of the model has already been investigated [8].
Although fairly complex, the big advantage of this model approach is that the behaviour of the spacecraft battery under any given current / power / temperature profile can be determined in order to tell in advance whether a proposed payload usage or failure scenario could be supported without entering safe mode.

\section{REFERENCES}

1. Ferretti, R Denis, M Moorhouse, A Dudley, G Ecale, E Rabenau, E Page, O Schulster, J \& Ormston, T (2006). Mars Express Power Subsystem In-flight Experience. SpaceOps 2006 Conference, Rome Italy AIAA-2006-5857

2. Dudley, G. De Roche, J Tonicello, F, \& Thwaite. C. (2005). Electrical/Thermal Model of a Sony 18650HC Li-Ion Cell, Proc. 7th European Space Power Conference (Ed. A. Wilson), ESA SP-589 (CD-ROM), European Space Agency, Noordwijk, The Netherlands.

3. Dudley, G. Thwaite, C. \& Walpole, A. (2006). Use of Electrical/Thermal Cell Model for Mars Express Battery Health Assessment. NASA Aerospace Battery Workshop, Huntsville USA

4. Dudley, G. Buckle, R. Hendel, B. Mattle, T. \& Spurrett, R. (2008). Ageing of Sony 18650 HC Cells in LEO Life Tests. Proc. 8th European Space Power Conference, (Eds. H. Lacoste \& L. Ouwehand) ESA SP-661 (CD-ROM), European Space Agency, Noordwijk, The Netherlands.

5. Dudley, G. (2014). Extension of Lithium Ion Cell Model to include Transient and Low-temperature behavior. Proc. 10th European Space Power Conference, (Ed. L. Ouwehand) ESA SP-719, European Space Agency, Noordwijk, The Netherlands.

\section{6. http://www.berkeleymadonna.com}

7. Sousa, B. \& van der Pols, CL. Breath in, breath out how healthy are the batteries on Mars and Venus Express?

http://www.spaceops2012.org/proceedings/docume nts/id1254584-Paper-001.pdf SpaceOps 2012 Conference, Stockholm, Sweden

8. Ringbeck, F. Marongiu, A. \& Becker, J. (2016). Report on Battery health status estimation based on modeling and advanced data processing techniques. Final report of ESTEC contract 4000114068/15/NL/HK with RWTH Aachen University 